\title{
Appearance of room-temperature ferromagnetism in $\mathrm{Cu}$-doped $\mathrm{TiO}_{2-\delta}$ films
}

\author{
S. Duhalde, M. F. Vignolo, and F. Golmar \\ Laboratorio de Ablación Láser, Depto. de Física, Facultad de Ingeniería, Universidad de Buenos Aires, Paseo Colón 850, \\ 1063 Buenos Aires, Argentina \\ C. Chiliotte \\ Laboratorio de Bajas Temperaturas, Facultad de Ciencias Exactas y Naturales, Universidad de Buenos Aires, \\ Intendente Güiraldes 2160, 1428 Buenos Aires, Argentina \\ C. E. Rodríguez Torres, L. A. Errico, A. F. Cabrera, M. Rentería, and F. H. Sánchez \\ Departamento de Física-Instituto de Física La Plata (CONICET), Facultad de Ciencias Exactas, Universidad Nacional \\ de La Plata, C.C. No. 67, 1900 La Plata, Argentina \\ M. Weissmann \\ Departamento de Física, Comisión Nacional de Energía Atómica, Av. del Libertador 8250, 1429 Buenos Aires, Argentina
} (Received 20 July 2005; revised manuscript received 19 September 2005; published 25 October 2005)

\begin{abstract}
We report here the unexpected observation of significant room-temperature ferromagnetism in a semiconductor doped with nonmagnetic impurities, $\mathrm{Cu}$-doped $\mathrm{TiO}_{2}$ thin films grown by pulsed laser deposition. The magnetic moment, calculated from the magnetization curves, resulted surprisingly large, about $1.5 \mu_{B}$ per $\mathrm{Cu}$ atom. A large magnetic moment was also obtained from ab initio calculations, but only if an oxygen vacancy in the nearest-neighbor shell of $\mathrm{Cu}$ was present. This result suggests that the role of oxygen vacancies is crucial for the appearance of ferromagnetism. The calculations also predict that $\mathrm{Cu}$ doping favors the formation of oxygen vacancies.
\end{abstract}

DOI: $10.1103 /$ PhysRevB.72.161313

PACS number(s): 75.70.-i, 75.50.Dd, 71.15.Mb, 85.75.-d

Integrating spin functionality into otherwise nonmagnetic materials has become a highly desirable goal in the last years. For example, dilute magnetic impurities in semiconductors produce materials appealing for spintronics (see, e.g., Refs. 1-3, and references therein). This is a rapidly developing research area, in which the electron spin plays an important role in addition to the usual charge degree of freedom. For their practical applications, ferromagnetic semiconductors are required to have a high Curie temperature $\left(T_{C}\right)$. While most of the dilute magnetic semiconductors (DMS) like Mn-doped GaAs (Ref. 4) have a $T_{C}$ much lower than room temperature, room-temperature ferromagnetism has been observed in some Mn-doped compounds such as $\mathrm{ZnO}^{5}$ Recently, Co-doped $\mathrm{TiO}_{2}$ thin films with the anatase structure were reported to be ferromagnetic even above $400 \mathrm{~K}$ with a magnetic moment of $0.32 \mu_{B}$ per Co atom. ${ }^{6}$ These results have motivated intensive experimental ${ }^{7-9}$ and theoretical ${ }^{10-14}$ studies on the structural and electronic properties of these materials. However, many questions remain open regarding the underlying microscopic mechanism of long-range magnetic order. Carrier-induced interaction between the magnetic atoms was first suggested as the important ingredient underlying ferromagnetism in III-V based DMS. ${ }^{15}$ Subsequent reports have raised concerns about the initially suggested intrinsic nature of ferromagnetism in these materials, due to the possibility of ferromagnetic metal clustering under different growth conditions. ${ }^{5,16}$ Furthermore, it has been suggested that the strong interaction between transition metal impurities and oxygen vacancies is crucial for the existence of high Curie temperature ferromagnetism. ${ }^{17-19}$ We believe our results shed light on this problem, suggesting a specific role for the oxygen vacancies and against the clustering hypothesis.

The original purpose of this work was to find out the role of oxygen deficiency in the origin and significance of ferromagnetism in doped $\mathrm{TiO}_{2}$ thin films. Thin films are used because they have a much greater surface or interface to volume ratio than single-crystals or polycrystalline ceramics, so the role of defects may be enhanced. On the other hand, we had previously found in Fe-doped $\mathrm{TiO}_{2}$ thin films analyzed by x-ray absorption near-edge and extended x-ray absorption fine structure spectroscopy that the magnetic signal increased when the number of oxygen vacancies around the impurity sites increased. ${ }^{20}$ In order to produce a similar concentration of vacancies in $\mathrm{TiO}_{2}$ thin films as in Fe-doped samples but without the magnetic ions, we studied $\mathrm{Cu}$-doped $\mathrm{TiO}_{2}$ films assuming that no magnetic signal would come from the dopant. Surprisingly, significant room temperature magnetic behavior, so strong to give a magnetization equivalent to $1.5 \mu_{B} / \mathrm{Cu}$, was found.

Thin films of approximately 10 at. $\% \mathrm{Cu}$-doped $\mathrm{TiO}_{2-\delta}$ were deposited on $\mathrm{LaAlO}_{3}$ (001) substrate (LAO) by pulsed laser deposition (PLD) using a Nd:yttrium-aluminum-garnet (YAG) laser operating at $266 \mathrm{~nm}$. The $\mathrm{TiO}_{2}: \mathrm{Cu}$ target was prepared from high purity $\mathrm{TiO}_{2}$ and metallic $\mathrm{Cu}$ powders in stoichiometric quantities. The powders were mixed for three minutes using a ball mill, then uniaxially pressed (200 MPa) into a disk, and finally sintered. The substrate temperature, laser energy density, oxygen pressure, and pulse repetition rate were $800{ }^{\circ} \mathrm{C}, 2 \mathrm{~J} / \mathrm{cm}^{2}, 20 \mathrm{~Pa}$, and $10 \mathrm{~Hz}$, respectively. After deposition the film was cooled down to room temperature in $2 \mathrm{~h}$ under $20 \mathrm{~Pa}$ oxygen atmosphere. The composition 


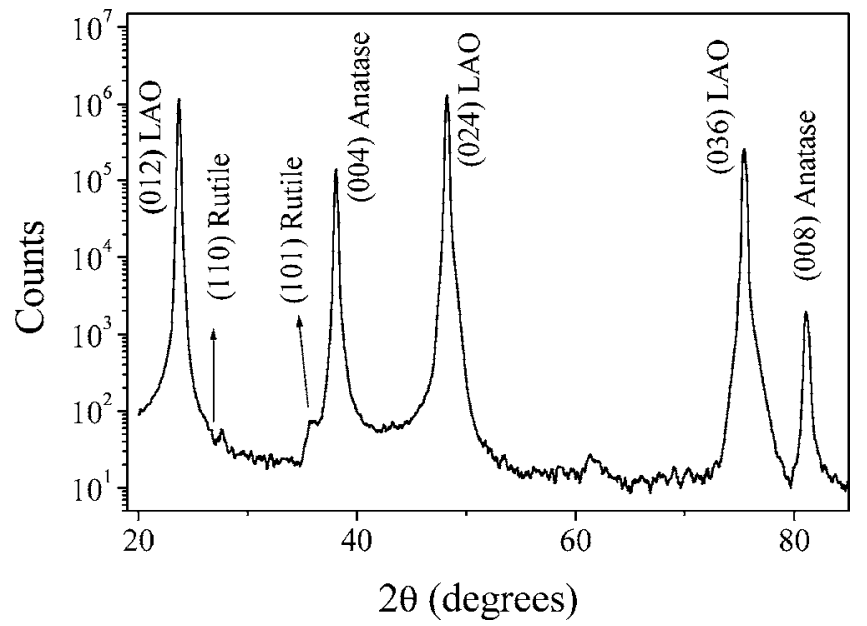

FIG. 1. X-ray diffraction pattern for $\mathrm{Cu}$-doped $\mathrm{TiO}_{2-\delta}$ film deposited by PLD on a $\mathrm{LaAlO}_{3}$ substrate.

was determined by energy dispersive x-ray analysis (EDX), and no contaminants were found within the accuracy of the method $(<1$ wt $\%)$. The crystalline structure was studied by $\mathrm{X}$-ray diffraction (XRD). Our films were transparent and strongly textured, showing only the (001) reflections of the anatase structure, although rutile reflections were also present but with much less intensity (Fig. 1). This strong difference between intensities does not directly reflect the relative amount of both phases, as our samples consist of a highly oriented anatase film [only (001) reflections with very large intensities] and polycrystalline rutile embedded in it [(110) and (101) reflections of low intensities]. The relative amount of the anatase and rutile polymorphs depends on the choice of substrate and on the growth conditions. The lattice mismatch between anatase $\mathrm{TiO}_{2}(001)$ and $\mathrm{LaAlO}_{3}(001)$ is only $0.26 \%$, much less than for rutile, so anatase is favored when deposition is performed on (001) LAO substrate. However, it has been shown ${ }^{21}$ that a slow growth rate is required to produce anatase single crystal films of high crystallographic quality, while growing at a higher rate results in polycrystalline films containing both anatase and rutile phases. Chambers et al. ${ }^{21}$ also found that the rutile nanocrystals nucleate within the evolving anatase film in orientations that maximize the lattice match between the two phases.

The measurements of magnetization $M$ as a function of the applied magnetic field $H$ were performed with a commercial vibrating sample magnetometer Lake Shore 7407 at room temperature, with the external field applied parallel and perpendicular to the plane of the film. After subtraction of the diamagnetic contribution due to $\mathrm{LaAlO}_{3}$ we obtained the result depicted in Fig. 2, where significant room-temperature magnetization is displayed. In order to quantify the magnetic parameters (saturation magnetization $M_{s}$, intrinsic coercivity $H_{c}$, and remanent magnetization $M_{r}$ ) we propose the following fitting function for the demagnetization data:

$$
M=M_{s}\left((2 / \pi)\left\{\arctan \left[\left(H+H_{c}\right) / H_{c}\right] \tan (\pi S / 2)\right\}\right)+\chi H,
$$

where $S=M_{r} / M_{s}$. The first term is the usual function used to represent a ferromagnetic hysteresis curve ${ }^{23}$ and the second

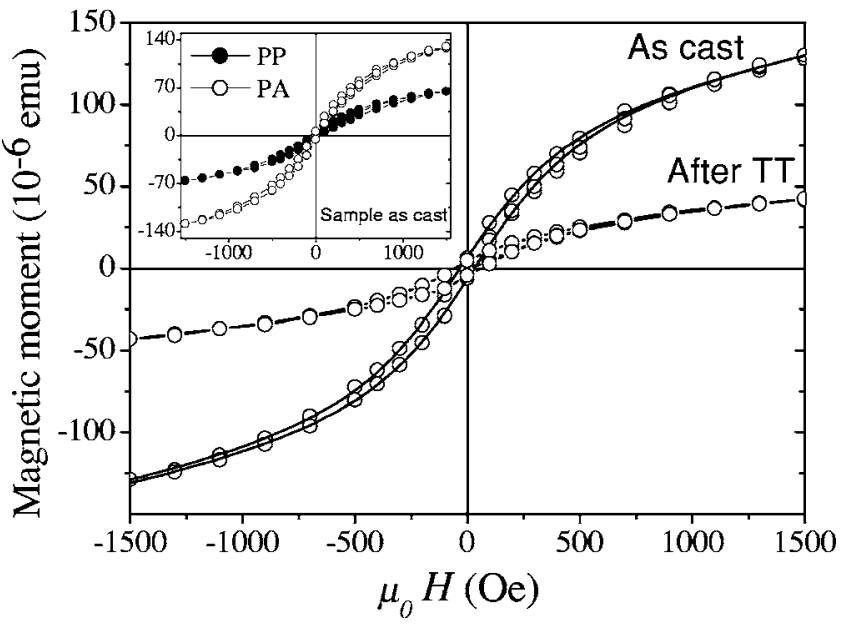

FIG. 2. Room-temperature hysteresis curve of the $\mathrm{Cu}$-doped $\mathrm{TiO}_{2-\delta}$ film (points) in the sample as cast and after a thermal treatment (TT) at $800{ }^{\circ} \mathrm{C}$ for $30 \mathrm{~min}$ in a oxygen-rich atmosphere. Solid lines are the best least-squares fits of Eq. (1) to the data. The inset compares the hysteresis loops measured with the external magnetic field applied parallel (PA) and perpendicular (PP) to the film plane for the as cast sample.

is a linear component representing a possible paramagnetic contribution. The parameters obtained are: $44_{8} \mathrm{emu} / \mathrm{cm}^{3}$, $2.5_{4} \mathrm{emu} / \mathrm{cm}^{3}$, and $35_{3}$ Oe for $M_{s}, M_{r}$, and $H_{c}$, respectively. From $M_{s}$, we estimate an atomic moment of $1.5 \mu_{B} / \mathrm{Cu}$, assuming 10 at. \% of $\mathrm{Cu}$ and a $1000 \AA$ film thickness.

For the interpretation of this unexpected experimental result we performed $a b$ initio calculations on doped $\mathrm{TiO}_{2}$, with and without oxygen vacancies, using the supercell (SC) method. As a first approximation we employed a small SC $\left(\mathrm{Ti}_{4} \mathrm{O}_{8}\right)$ consisting of two unit cells of the rutile structure stacked along the $c$ axis. One $\mathrm{Ti}$ atom was replaced by $\mathrm{Cu}$, giving a rather large impurity concentration compared with the experimental one. However, the appearance of a magnetic moment in some cases was also quite surprising and interesting to relate with the experiments. The rutile structure was chosen for the calculations because it gives a more uniform distribution of defects than the anatase structure when the SC is small. This leads to a more realistic approximation of the structural distortions induced by the impurity and the vacancy in the host lattice. The calculations were performed with the $a b$ initio full-potential linearized-augmented-planewave method (FP-LAPW) in a scalar relativistic version, as implemented in the WIEN2K code. ${ }^{24}$ We used the local spin density approximation (LSDA) and the exchange-correlation potential given by Perdew and Wang. ${ }^{25}$ The cutoff criterion was $\mathrm{R}_{m t} \mathrm{~K}_{M A X}=7, \mathrm{R}_{m t}$ being the smallest muffin tin radius and $\mathrm{K}_{M A X}$ the largest wave number of the basis set. The number of $k$ points was increased until convergence was reached. We also employed, especially for structural minimizations, the SIESTA code ${ }^{26}$ that uses a linear combination of numerical real-space atomic orbitals as basis set and normconserving pseudopotentials. We proved using WIEN2K that the relaxed structures predicted by SIESTA have lower energies than the unrelaxed ones and that both methods give similar magnetic moments. 
The SC used for these calculations is tetragonal $(a=b$ $\left.=4.5845_{1} \AA, c^{\prime}=2 c=5.9066_{2} \AA\right)^{22}$ and contains $4 \mathrm{Ti}$ atoms at $[(0,0,0) ;(1 / 2,1 / 2,1 / 4) ; R]$ and $8 \mathrm{O}$ atoms at $[(u, u, 0)$; $(1 / 2+u, 1 / 2-u, 1 / 4) ; R]$ with $u=0.3049 .{ }^{22}$ The notation $R$ indicates that the group of coordinates should be repeated adding $(0,0,1 / 2)$. In this structure, the Ti atoms are surrounded by a slightly distorted oxygen octahedron with a rectangular basal plane of four oxygen atoms with distances to $\mathrm{Ti}(1.94 \AA)$ slightly shorter than those at the vertex $(1.98$ $\AA$ ).

For the case of $\mathrm{Ti}_{3} \mathrm{CuO}_{8}$, the system without oxygen vacancies, we found that the presence of $\mathrm{Cu}$ induces a small distortion of the host lattice, mainly in the six oxygen nearest-neighbors $\left(\mathrm{O}_{N N}\right)$ of the $\mathrm{Cu}$ impurity. The $\mathrm{Cu}-\mathrm{O}_{N N}$ distances are enlarged from 1.94 and $1.98 \AA$ to 1.96 and 2.01 $\AA$, respectively. However, no magnetism was found, which suggests that the experimentally observed magnetic moment cannot be due only to the presence of $\mathrm{Cu}$ impurities.

In order to analyze the effect of oxygen vacancies in doped $\mathrm{TiO}_{2}$ we first studied the system $\mathrm{Ti}_{4} \mathrm{O}_{7}$, taking into account the structural distortions produced by the vacancies. These are much larger than those found in $\mathrm{Ti}_{3} \mathrm{CuO}_{8}$, as some of the Ti atoms are displaced as much as $0.2 \AA$. We found that for this system there are two possible degenerate solutions, one is nonmagnetic and the other has a magnetic moment of $1.1 \mu_{B}$ per SC, due to polarization of the Ti atoms. It is worth mentioning that the magnetic phase appears only when structural relaxation is taken into account. This result anticipates that oxygen vacancies could play an important role in the magnetic behavior observed in the $\mathrm{TiO}_{2}: \mathrm{Cu}$ films. The question that arises is why the magnetic phase is not observed in bulk rutile $\mathrm{TiO}_{2}$ without impurities. A possible explanation for this is that the concentration of intrinsic vacancies formed in equilibrium is not enough to generate magnetism at the temperature considered. Calculations with a larger unit cell and only one vacancy, both in rutile and anatase structures, were studied and a smaller magnetic moment was obtained for the rutile structure and none for the anatase structure. So it seems that the appearance of a magnetic moment may depend on the vacancy concentration and on the structure. An experimental observation of magnetism in an undoped nonmagnetic oxide has been recently observed experimentally and has been attributed to vacancies also. ${ }^{27}$

Finally, we studied the oxygen deficient $\mathrm{Cu}$-doped $\mathrm{TiO}_{2}$ systems $\left(\mathrm{Ti}_{3} \mathrm{CuO}_{7}\right)$. We considered the three possibilities for removing an oxygen atom from the $\mathrm{SC}$ : (i) from the Ticontained octahedron, (ii) from the $\mathrm{Cu}$-contained octahedron removing a basal $\mathrm{O}$ atom, and (iii) from the $\mathrm{Cu}$-contained octahedron removing a vertex $\mathrm{O}$ atom. Comparing the total energies of the three cases we found that the oxygen vacancies near $\mathrm{Cu}$ are more stable than those near the Ti site. A similar result was found by Weng et al. ${ }^{13}$ for Co in oxygendeficient $\mathrm{TiO}_{2}$.

The calculations also predict that the energy required to form an oxygen vacancy decreases from $10 \mathrm{eV}$ in the undoped system to $4 \mathrm{eV}$ in the case it is near a $\mathrm{Cu}$ impurity. Also, the energy required to replace a $\mathrm{Ti}$ atom by a $\mathrm{Cu}$ atom is reduced by $5 \mathrm{eV}$ when there is an oxygen vacancy in the SC. We therefore predict that $\mathrm{Cu}$ doped systems will have

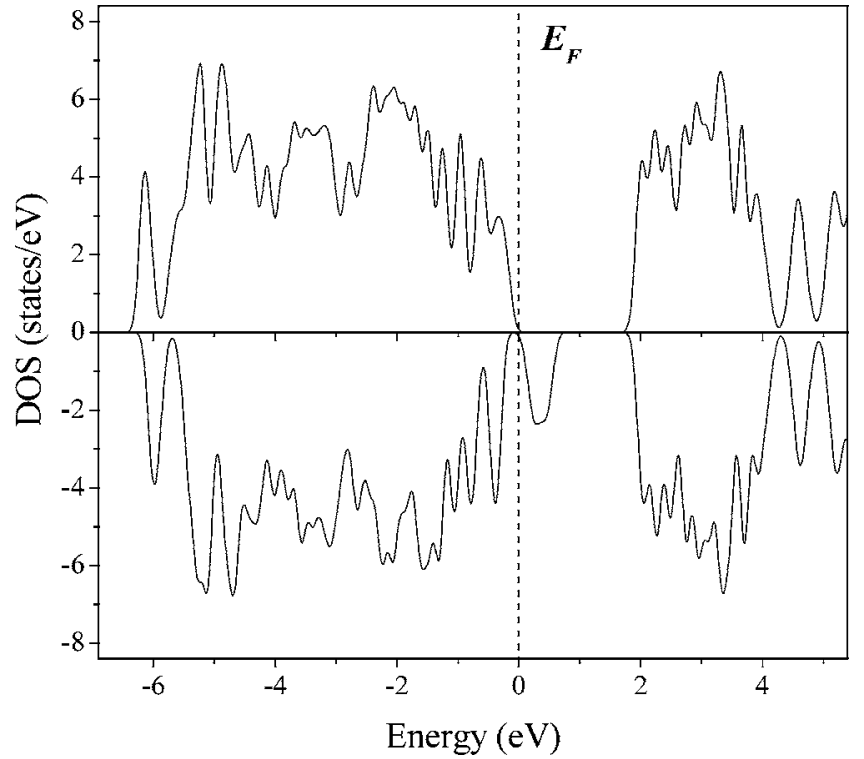

FIG. 3. Density of states of $\mathrm{Ti}_{3} \mathrm{CuO}_{7}$, with the oxygen vacancy near $\mathrm{Cu}$. Positive values are for spin up and the negative ones for spin down. Energies are referred to the Fermi level, $E_{F}$. The minority spin feature inside the gap but close to $E_{F}$ is $50 \%$ of $\mathrm{Cu} 3 d$ and $50 \%$ of oxygen and $\mathrm{Ti}$ character.

more vacancies than the undoped ones, and that oxygen vacancies will tend to be close to the impurities. We also found that the energy required to form two vacancies near a $\mathrm{Cu}$ impurity is larger than that necessary to create one vacancy in the first shell of neighbors of two different $\mathrm{Cu}$ impurities. For this reason, we discard the formation of vacancy clusters around $\mathrm{Cu}$ atoms.

Concerning the magnetic properties of the system $\mathrm{Ti}_{3} \mathrm{CuO}_{7}$, we found that the magnetic solution has lower energy than the nonmagnetic one, with a magnetic moment of $1.0 \mu_{B}$ per $\mathrm{SC}$, due to polarization of both $\mathrm{Cu}$ and $\mathrm{O}_{N N}$ atoms. The value of the magnetic moment is nearly independent of vacancy location and of structural relaxation. This result indicates that there is magnetism in $\mathrm{Cu}$-doped $\mathrm{TiO}_{2}$, but as we have considered only one SC size (and therefore only one impurity concentration) we cannot compare its value with the experimental magnetic moment per $\mathrm{Cu}$ atom. To do this we should average over three different concentrations: that of $\mathrm{Cu}$, that of vacancies, and the relative amount of rutile and anatase phases. As a first step in this direction we performed a few calculations with a larger SC containing 48 atoms. The dimensions of this SC are $a^{\prime}=b^{\prime}=2 a=2 b$ $=9.169 \AA, c^{\prime}=2 c=5.907 \AA$. In particular, for the case of one $\mathrm{Cu}$ atom with a neighbor oxygen vacancy in the SC we obtained a magnetic moment of approximately $1.0 \mu_{B}$, which validates the results obtained for the smaller SC. A similar result was found for the anatase phase.

Figure 3 shows a calculated density of states of the system $\mathrm{Ti}_{3} \mathrm{CuO}_{7}$, which is small gap semiconductor or a semimetal. However, if the number of vacancies were slightly larger than that of the $\mathrm{Cu}$ impurities, the Fermi level would move into the minority spin feature close to it. In this case the system would be magnetic and at the same time halfmetallic, with carriers of only one spin type; this could be 
very interesting for spintronics. A similar idea, with excess vacancies responsible for ferromagnetism, was proposed recently. ${ }^{28}$

In the previous calculations a ferromagnetic alignment of the $\mathrm{Cu}$ spins was assumed, but we also verified that the pairs $\mathrm{Cu}-\mathrm{O}_{N N}$ vacancy do not couple antiferromagnetically. This last calculation was performed for a few particular cases, as the number of possible distributions of impurities and vacancies is large and so is the supercell required for that calculation.

In order to verify the crucial role played by the oxygen vacancies in the origin of ferromagnetism, we performed a "reverse experiment": in order to reduce the oxygen vacancies, the $\mathrm{TiO}_{2}$ film was heated at $800{ }^{\circ} \mathrm{C}$ for $30 \mathrm{~min}$ in an oxygen-rich atmosphere. While the XRD spectra show no changes, the measurements of $M$ as a function of $H$ after the thermal treatment show a drastic reduction of the magnetic signal (see Fig. 2), in agreement with the $a b$ initio predictions.
In conclusion, we have observed an unexpected significant room temperature magnetic behavior in $\mathrm{Cu}$-doped $\mathrm{TiO}_{2-\delta}$ films, so strong to give $1.5 \mu_{B}$ per $\mathrm{Cu}$ atom. This experimental result indicates that magnetic ions are not essential to obtain this effect and also that is not due to impurity clustering. Ab initio calculations on bulk $\mathrm{Cu}$-doped $\mathrm{TiO}_{2}$, with and without oxygen vacancies, predict a magnetic moment of $1.0 \mu_{B}$ for a supercell containing one $\mathrm{Cu}$ impurity and a neighbor oxygen vacancy, but no magnetic moment if the oxygen vacancy is absent. The calculations also predict a lower vacancy formation energy when there are $\mathrm{Cu}$ impurities. Therefore, it seems that oxygen vacancies play a crucial role for the appearance of magnetism and that one effect of doping is to increase their number. Both experimental and theoretical work is now in progress.

This work was partially supported by ANPCyT, PICT98 03-03727, CONICET, Fund. Antorchas, Argentina, and TWAS, Italy, RGA 97-057. We thank R. Weht for his assistance with the SIESTA code.
${ }^{1}$ H. Ohno, Science 281, 951 (1998).

${ }^{2}$ T. Dietl, H. Ohno, F. Matsukura, J. Cibert, and D. Ferrand, Science 287, 1019 (2000).

${ }^{3}$ T. Fukumura, Y. Yamada, H. Toyosaki, T. Hasegawa, H. Koinuma, and M. Kawasaki, Appl. Surf. Sci. 223, 62 (2004).

${ }^{4}$ H. Ohno, A. Shen, F. Matsukura, A. Oiwa, A. Endo, S. Katsumoto, and Y. Iye, Appl. Phys. Lett. 69, 363 (1996).

${ }^{5}$ P. Sharma, A. Gupta, K. V. Rao, F. J. Owens, R. Sharma, R. Ahuja, J. M. Osorio Guillen, B. Johansson, and G. A. Gehring, Nat. Mater. 24, 673 (2003)

${ }^{6}$ Y. Matsumoto, M. Murakami, T. Shono, T. Hasegawa, T. Fukumura, M. Kawasaki, P. Ahmet, T. Chikyow, S. Y. Koshihara, and H. Koinuma, Science 291, 854 (2001).

${ }^{7}$ W. K. Park, R. J. Ortega-Hertogs, J. S. Moodera, A. Punnoose, and M. S. Seehra, J. Appl. Phys. 91, 8093 (2002).

${ }^{8}$ S. A. Chambers, S. Thevuthasan, R. F. C. Farrow, R. F. Marks, J. U. Thiele, L. Folks, M. G. Samant, A. J. Kellock, N. Ruzycki, D. L. Ederer, and U. Diebold, Appl. Phys. Lett. 79, 3467 (2001).

${ }^{9}$ Y. L. Soo, G. Kioseoglou, S. Kim, and Y. H. Kao, P. Sujatha Devi, J. Parise, R. J. Gambino, and P. I. Gouma, Appl. Phys. Lett. 81, 655 (2002).

${ }^{10}$ M. S. Park, S. K. Kwon, and B. I. Min, Phys. Rev. B 65, 161201(R) (2002).

${ }^{11}$ Z. Yang, G. Liu, and R. Wu, Phys. Rev. B 67, 060402(R) (2003).

${ }^{12}$ W. T. Geng and K. S. Kim, Phys. Rev. B 68, 125203 (2003).

${ }^{13}$ H. Weng, X. Yang, J. Dong, H. Mizuseki, M. Kawasaki, and Y. Kawasoe, Phys. Rev. B 69, 125219 (2004).

${ }^{14}$ L. A. Errico, M. Weissmann, and M. Rentería, Phys. Status Solidi B 241, 2399 (2004); L. A. Errico, M. Weissmann, and M. Rentería, Physica B 354, 338 (2004).

${ }^{15}$ H. Munekata, H. Ohno, S. von Molnar, A. Segmüller, L. L. Chang, and L. Esaki, Phys. Rev. Lett. 63, 1849 (1989).
${ }^{16}$ S. R. Shinde, S. B. Ogale, S. Das Sarma, J. R. Simpson, H. D. Drew, S. E. Lofland, C. Lanci, J. P. Buban, N. D. Browning, V. N. Kulkarni, J. Higgins, R. P. Sharma, R. L. Greene, and T. Venkatesan, Phys. Rev. B 67, 115211 (2003).

${ }^{17}$ R. Suryanarayanan, V. M. Naik, P. Kharel, P. Talagala, and R. Naik, Solid State Commun. 133, 439 (2005); R. Suryanarayanan, V. M. Naik, P. Kharel, P. Talagala, and R. Naik, J. Phys.: Condens. Matter 17, 755 (2005).

${ }^{18}$ M. Venkatesan, C. B. Fitzgerald, J. G. Lunney, and J. M. D. Coey, Phys. Rev. Lett. 93, 177206 (2004)

${ }^{19}$ J. M. D. Coey, M. Venkatesan, and C. B. Fitzgerald, Nat. Mater. 4, 173 (2005).

${ }^{20}$ C. E. Rodríguez Torres, A. F. Cabrera, S. Duhalde, and F. H. Sánchez (unpublished).

${ }^{21}$ S. A. Chambers, C. M. Wang, S. Thevuthasan, T. Droubay, D. E. McCready, A. S. Lea, V. Shutthanandan, and C. F. Windisch, Jr., Thin Solid Films 418, 197 (2002).

${ }^{22}$ J. Hill and C. J. Howard, J. Appl. Crystallogr. 20, 467 (1987).

${ }^{23}$ M. B. Stearns and Y. Cheng, J. Appl. Phys. 75, 6894 (1994).

${ }^{24}$ P. Blaha, K. Schwarz, G. K. H. Madsen, D. Kvasnicka, and J. Luitz, WIEN2K, An Augmented Plane Wave + Local Orbitals Program for Calculating Crystal Properties (K. Schwarz, Techn. Univ. Wien, Austria, 2001).

${ }^{25}$ J. P. Perdew and Y. Wang, Phys. Rev. B 45, 13244 (1992).

${ }^{26}$ P. Ordejón, E. Artacho, and J. M. Soler, Phys. Rev. B 53, R10441 (1996); D. Sánchez-Portal, P. Ordejón, E. Artacho, and J. M. Soler, Int. J. Quantum Chem. 65, 453 (1997).

${ }^{27}$ M. Venkatesan, C. B. Fitzgerald, and J. M. D. Coey, Nature 430, 630 (2004)

${ }^{28}$ A. C. Tuan, J. D. Bryan, A. Pakhomov, V. Shutthanandan, S. Thevuthasan, D. McCready, D. Gaspar, M. Engelhard, J. W. Rogers, Jr., K. Krishnan, D. R. Gamelin, and S. A. Chambers, Phys. Rev. B 70, 054424 (2004). 